\title{
A NOTE ON CALDERÓN-ZYGMUND SINGULAR INTEGRAL CONVOLUTION OPERATORS
}

\author{
JOAQUIM BRUNA AND BORIS KORENBLUM
}

The purpose of this note is to show that the notion of weak maximal function introduced in [1] (see also [4], where a similar notion is considered) can be used to obtain some new information on the Calderón-Zygmund singular integral convolution operator.

We will follow the notations of $[\mathbf{3}]$. Let $K$ be a kernel in $\mathbf{R}^{n}$ of class $C^{1}$ outside the origin satisfying

$$
\begin{gathered}
|K(x)| \leq C|x|^{-n}, \\
|\nabla K(x)| \leq C|x|^{-n-1} .
\end{gathered}
$$

For $\varepsilon>0$ and $f \in L^{p}\left(\mathbf{R}^{n}\right), 1 \leq p<\infty$, set

$$
T_{\varepsilon}(f)(x)=\int_{|y| \geq \varepsilon} f(x-y) K(y) d y
$$

and

$$
T(f)(x)=\lim _{\varepsilon \rightarrow 0} T_{\varepsilon}(f)(x), \quad T^{*}(f)(x)=\sup _{\varepsilon>0}\left|T_{\varepsilon}(f)(x)\right| .
$$

We will assume that $K$ satisfies the usual properties ensuring that the mapping $f \mapsto T^{*}(f)$ is of weak type $(1,1)$ and that $T(f)(x)$ makes sense for a.e. $x$.

The notation $L^{1, \infty}$ will stand for the space of weak $L^{1}$ functions, and if $\varphi \in L^{1, \infty}$ and $B$ is a ball we write

$$
\|\varphi\|_{1, \infty}^{B}=\sup _{\delta>0} \delta m(\{x \in B:|\varphi(x)|>\delta\})
$$

for the weak $L^{1}$ "norm" of $\varphi$ on $B$. If $B=\mathbf{R}^{n}$, we simply write $\|\varphi\|_{1, \infty}$.

The weak maximal function introduced in [1] is defined for $\varphi \in L^{1, \infty}$ by

$$
M_{w} \varphi(x)=\sup _{x \in B} \frac{\|\varphi\|_{1, \infty}^{B}}{m(B)},
$$

the supremum being taken over all balls centered at $x$. The notation $M_{w}^{m} \varphi$ stands for the function obtained by applying $m$ times the operator $M_{w}$, whenever this makes sense. In [1] it was already pointed out that for any $m$ there is a $\varphi \in L^{1, \infty}$ such that $M_{w}^{j} \varphi \in L^{1, \infty}$ for $j=1, \ldots, m$ but $M_{w}^{m+1} \varphi \notin L^{1, \infty}$. However, for $\varphi=T f, f \in L^{1}$, the following holds:

Received by the editors May 20, 1986 and, in revised form, May 31, 1986.

1980 Mathematics Subject Classification (1985 Revision). Primary 42B20.

First author supported by grant No. 1593/82 of the Comisión Asesora de Investigación Cientifica y Técnica, Madrid.

Second author supported by NSF grant DMS-8600699. 
THEOREM. If $T$ is as above, $M_{w}^{m} T^{*} f \in L^{1, \infty}$ for all $f \in L^{1}$ and all $m \in N$, and $\left\|M_{w}^{m} T^{*} f\right\|_{1, \infty}$ grows as a geometric progression. Hence the same is true for $T f$.

As in [1], the motivation for this research is the following

QUESTION. What is the necessary and sufficient condition on a nonnegative function $\varphi$ for the existence of $f \in L^{1}$ such that

$$
\varphi \leq|T f| \text { a.e.? }
$$

In other words, what is the precise description of the magnitude of $T f$ ? The theorem gives a necessary condition stronger than $\varphi \in L^{1, \infty}$, namely,

$$
\left\|M_{w}^{m} \varphi\right\|_{1, \infty} \leq C_{1} C_{2}^{m}
$$

but, as shown in the last section of [1], this condition is not sufficient (see $\S 5$ of [1] for other remarks concerning this question).

PROOF OF THE THEOREM. Let us first remark that the corresponding result with $T f$ replaced by the Hardy-Littlewood maximal function $M f$ is also true. In fact in this case something more precise is true, namely

$$
M_{w} M f(x) \leq C M f(x)
$$

for some constant $C=C(n)$. This is shown in [1, pp. 9-10], and it is also implicit in [2].

In fact, our proof of the theorem will be a consequence of something similar to (3). We will show that

$$
M_{w} T^{*} f \leq C\left\{T^{*} f+M f\right\} .
$$

Together with (3) this will give

$$
M_{w}^{m} T^{*} f \leq C^{m}\left\{T^{*} f+M f\right\}
$$

(note that $M_{w}(\varphi+\psi) \leq 2\left(M_{w} \varphi+M_{w} \psi\right)$ ), which clearly implies the theorem.

In order to prove (4), fix $x$ and let $B$ be a ball centered at $x$. Let $2 B$ denote the ball having the same center as $x$ and twice the radius and set

$$
f_{1}=f \chi_{2 B}, \quad f_{2}=f-f_{1} .
$$

Then $T^{*} f \leq T^{*} f_{1}+T^{*} f_{2}$ and

$$
\left\|T^{*} f\right\|_{1, \infty}^{B} \leq 2\left(\left\|T^{*} f_{1}\right\|_{1, \infty}^{B}+\left\|T^{*} f_{2}\right\|_{1, \infty}^{B}\right) .
$$

Since $T^{*}$ satisfies a weak $(1,1)$ estimate, we have

$$
\begin{aligned}
\left\|T^{*} f_{1}\right\|_{1, \infty}^{B} & \leq\left\|T^{*} f_{1}\right\|_{1, \infty} \\
& \leq C\left\|f_{1}\right\|_{1}=C \int_{2 B}|f(y)| d y \leq C m(B) M f(x) .
\end{aligned}
$$

Now we will prove that for $z \in B$

$$
|| T^{*} f_{2}(z) \mid \leq C\left(T^{*} f(x)+M f(x)\right) .
$$

This implies

$$
\left\|T^{*} f_{2}\right\|_{1, \infty}^{B} \leq C m(B)\left(T^{*} f(x)+M f(x)\right),
$$


and together with (5) this gives

$$
\left\|T^{*} f\right\|_{1, \infty}^{B} \leq C m(B)\left(T^{*} f(x)+M f(x)\right),
$$

which is (4).

For (6) we have to prove that for any $\varepsilon>r, r$ being the radius of $B$,

$$
\left|T_{\varepsilon} f_{2}(z)\right| \leq C\left(T^{*} f(x)+M f(x)\right) .
$$

Now

$$
T_{\varepsilon} f_{2}(z)=\int_{\substack{\mathbf{R}^{n} \backslash 2 B \\|y-z| \geq \varepsilon}} f(y) K(z-y) d y .
$$

If $\delta=\varepsilon+r$, it is clear that the contribution in this integral of $B_{0}=\delta B / r$, the ball centered at $x$ of radius $\delta$, is dominated (using (1)) by

$$
C \varepsilon^{-n} \int_{B_{0}}|f(y)| d y \leq C M f(x) .
$$

It remains to estimate

$$
I \stackrel{\text { def }}{=} \int_{|y-x|>\delta} f(y) K(z-y) d y .
$$

This is compared with $T_{\delta} f(x)$ in the usual way:

$$
I-T_{\delta} f(x)=\int_{|y-x|>\delta} f(y)\{K(z-y)-K(x-y)\} d y .
$$

Using (2) we obtain

$$
\left|I-T_{\delta} f(z)\right| \leq C|z-x| \int_{|y-x|>\delta}|f(y)| \frac{d y}{|y-x|^{n+1}},
$$

and it is well known that this is in turn dominated by $M f(x)$. Therefore we have proved that

$$
\left|T_{\varepsilon} f_{2}(z)\right| \leq C\left(\left|T_{\delta} f(x)\right|+M f(x)\right)
$$

which yields (7) and finishes the proof of the theorem.

REMARK. S. Drury (private communication) has independently generalized some results of [ 1 ] proving that $M_{w} T f \in L^{1, \infty}$, i.e. the case $m=1$ of the Theorem. He replaces the condition (1) and (2) by the so-called Hörmander condition (see $\left[3\right.$, p. 34 , condition $\left.\left(2^{\prime}\right)\right]$ ).

\section{REFERENCES}

1. J. Bruna and B. Korenblum, On Kolmogorov's theorem, the Hardy-Littlewood maximal function and the radial maximal function, preprint.

2. R. R. Coifman and R. Rochberg, Another characterization of BMO, Proc. Amer. Math. Soc. 79 (1980), 249-254.

3. E. M. Stein, Singular integrals and differentiability properties of functions, Princeton Univ. Press, Princeton, N.J., 1970.

4. - Editor's note: The differentiability of functions in $R^{n}$, Ann. of Math. (2) 133 (1981), 383-385.

Department de MATemàtiques, Universitat Autònoma de Barcelona, Bellaterra, Barcelona, SPAin

Department of Mathematics and Statistics, State UNiVersity OF NEW YORK, ALBANY, NEW YORK 12222 